\title{
EFFECTIVENESS OF INTERDISCIPLINARY HEALTH EDUCATION PROGRAMS FOR INDIVIDUALS WITH FIBROMYALGIA: A SYSTEMATIC REVIEW
}

Mateus Dias Antunes ${ }^{1, *}$, Leticia Assis Couto ${ }^{1}$, Sonia Maria Marques Gomes Bertolini², Felipe Cayres Nogueira da Rocha Loures², Ana Carolina Basso Schmitt ${ }^{1}$, Amélia Pasqual Marques ${ }^{1}$

1. Universidade de São Paulo, São Paulo, SP, Brazil. 2. Universidade Cesumar, Maringá, PR, Brazil.

*Corresponding author: mateusantunes@usp.br

\section{BACKGROUND}

The reviewed recommendations of the European League Against Rheumatism (EULAR) for fibromyalgia management indicate that the initial strategy should focus on patient education and nonpharmacological interventions. Health education is one of the main items to enable health promotion at primary health care in Brazil. The aim of this study was to verify the effectiveness of interdisciplinary health education programs for individuals with fibromyalgia.

\section{MATERIAL AND METHODS}

This systematic review followed the Preferred Reporting Items for Systematic Reviews and Meta-Analyses (PRISMA) checklist. The study was registered at the International Prospective Register of Systematic Reviews (PROSPERO) under no. CRD4201913228. A search was conducted at the following databases: SciELO, LILACS, MEDLINE, Scopus, Web of Knowledge ISI, PEDro, Embase, CINAHL, Cochrane Library, and SPORTDiscus. The descriptors used were "Fibromyalgia" and "Health Education". Clinical trials published between 1990 and 2019 were selected. The Jadad Quality Scale and the Cochrane Risk-of-Bias Tool were used to evaluate the risk of bias and the methodological quality of the clinical trials.

\section{RESULTS}

The search found 2887 articles, and two studies were selected for the present review after application of the exclusion criteria. One study was conducted in Brazil and the other in Spain. It was observed that one interdisciplinary health education program improved pain and quality of life in individuals with fibromyalgia. Both studies conducted the interventions through lectures and group activities. In particular, the topics most frequently approached at the interdisciplinary health education programs were general information about fibromyalgia, body practices, physical activities, and pharmacological approaches. Regarding intervention duration, a difference was observed between the two studies. Intervention varied from 4 (28 days) to 11 weeks (77 days). There was no consistency regarding the analyzed variables and the evaluation tools. The evidence quality showed that one study presented low risk of bias and the other presented high risk of bias. Regarding the finding of low methodological quality, double-blinding was the least satisfying criterion.

\section{CONCLUSION}

An interdisciplinary health education program can improve pain and quality of life in people with fibromyalgia; however, evidence shows low methodological quality. Researchers should consider the results of this systematic review when suggesting new interdisciplinary health education programs for people with fibromyalgia.

\section{ACKNOWLEDGMENTS}

This study was financed in part by the Coordenação de Aperfeiçoamento de Pessoal de Nível Superior - Brasil (CAPES) - Finance Code 001. 